\title{
VAZÕES MÁXIMAS E MÍNIMAS PARA BACIAS HIDROGRÁFICAS DA REGIÃO ALTO RIO GRANDE, MG
}

\author{
Maximum and minimum discharges for Alto Rio Grande region basins, \\ Minas Gerais state, Brazil
}

\author{
Carlos Rogério de Mello', Marcelo Ribeiro Viola², Samuel Beskow ${ }^{3}$
}

\begin{abstract}
RESUMO
Vazões máximas são grandezas hidrológicas aplicadas a projetos de obras hidráulicas e vazões mínimas são utilizadas para a avaliação das disponibilidades hídricas em bacias hidrográficas e comportamento do escoamento subterrâneo. Neste estudo, objetivouse à construção de intervalos de confiança estatísticos para vazões máximas e mínimas diárias anuais e sua relação com as características fisiográficas das 6 maiores bacias hidrográficas da região Alto Rio Grande à montante da represa da UHE-Camargos/CEMIG. As distribuições de probabilidades Gumbel e Gama foram aplicadas, respectivamente, para séries históricas de vazões máximas e mínimas, utilizando os estimadores de Máxima Verossimilhança. Os intervalos de confiança constituem-se em uma importante ferramenta para o melhor entendimento e estimativa das vazões, sendo influenciado pelas características geológicas das bacias. Com base nos mesmos, verificou-se que a região Alto Rio Grande possui duas áreas distintas: a primeira, abrangendo as bacias Aiuruoca, Carvalhos e Bom Jardim, que apresentaram as maiores vazões máximas e mínimas, significando potencialidade para cheias mais significativas e maiores disponibilidades hídricas; a segunda, associada às bacias F. Laranjeiras, Madre de Deus e Andrelândia, que apresentaram as menores disponibilidades hídricas.
\end{abstract}

Termos para indexação: Intervalos de confiança, rendimentos específicos, hidrologia estatística.

\begin{abstract}
Maximum discharges are applied to hydraulic structure design and minimum discharges are used to characterize water availability in hydrographic basins and subterranean flow. This study is aimed at estimating the confidence statistical intervals for maximum and minimum annual discharges and their relationship wih the physical characteristics of basins in the Alto Rio Grande Region, State of Minas Gerais. The study was developed for the six (6) greatest Alto Rio Grande Region basins at upstream of the UHE-Camargos/CEMIG reservoir. Gumbel and Gama probability distribution models were applied to the maximum and minimum long-term discharges respectively, using Maximum Likelihood estimators. Confidence intervals are important tools for better understand and estimation of water flows, being influenced by the geological characteristics of the basins. Based on the results, we could distinguish two hydrological systems: Aiuruoca, Carvalhos, and Bom Jardim basins, which have produced the greatest maximum and minimum discharges, which means they are more prone to floods, and have more water availability; and F. Laranjeiras, Madre de Deus and Andrelandia basins, which presented smaller water availability.
\end{abstract}

Index terms: Confidence intervals, specific discharges, statistical hydrology.

(Recebido em 20 de agosto de 2008 e aprovado em 24 de abril de 2009)

\section{INTRODUÇÃO}

O gerenciamento de recursos hídricos vem sendo exigido por lei, no Brasil, com o objetivo de disciplinar e melhorar o manejo dos mesmos, evitando conflitos e reduzindo custos, bem como consequências danosas nos seus aspectos qualitativos e quantitativos.

Neste contexto, acentua-se a importância do conhecimento hidrológico, o qual possibilita a determinação de valores adequados de vazões de referência para disponibilidade hídrica. Quando se dispõe de séries históricas de vazão de cursos d'água, tem-se a necessidade de trabalhálas adequadamente por meio de análises probabilísticas e / ou estocásticas (Haan, 2002; Douglas \& Vogel, 2006).
De acordo com Martins \& Clarke (1993), a construção de intervalos de confiança para os estimadores de Máxima Verossimilhança é de fundamental importância para melhoria das estimativas de vazões. De acordo com estes autores, a cobertura desses intervalos é, consideravelmente, mais precisa que os intervalos gerados com base no teorema central do limite, cuja construção é bastante trabalhosa e menos confiável para distribuições assintóticas. Para sua estimativa, aplicam-se métodos de simulação, como a reamostragem de Jackniff e Monte-Carlo (Casella \& Berger, 1990; Beijo et al., 2005).

Katz et al. (2002) e Queiroz \& Chaudhry (2006) relatam que variáveis hidrológicas com distribuição assintótica, como vazões máximas e mínimas, devem ser

1 Universidade Federal de Lavras/UFLA - Departamento de Engenharia/DEG - Cx.P. 3037 - 37200-000 - Lavras, MG - crmello@ufla.br
2Universidade Federal de Lavras/UFLA - Departamento de Engenharia/DEG - Lavras, MG

${ }^{3}$ National Soil Erosion Research Lab - Purdue University, Indiana - USA

Ciênc. agrotec., Lavras, v. 34, n. 2, p. 494-502, mar./abr., 2010 
adequadamente tratadas do ponto de vista probabilístico, com ênfase nas metodologias de maior precisão, especialmente, da máxima verossimilhança e dos momentos LH, os quais correspondem a momentos ponderados por probabilidades, sendo uma generalização de momentos de combinações lineares de estatísticas de ordem superior. No entanto, de acordo com George (2007), é essencial que os resultados obtidos pela simulação estocástica encontrem embasamento nas características físicas e geológicas das bacias de drenagem, especialmente para identificação de regiões hidrologicamente homogêneas.

Mello et al. (2002) e Maki et al. (2007) comentam que o comportamento hidrológico de bacias hidrográficas é resultado, especialmente, das características pedológicas das mesmas, como capacidade de infiltração e de retenção de água, influenciando na geração do deflúvio e recarga de aquíferos. Nesta mesma linha, Araújo (2006), estudando características pedológicas mais importantes para recarga subterrânea e manutenção do escoamento subterrâneo nesta região, comenta que as características geológicas são primordiais para o entendimento do comportamento de vazões mínimas, pois determinam as condições de drenagem do aquífero para os cursos d'água.

Dentro deste contexto, objetivou-se com este trabalho, estimar os intervalos de confiança para vazões máximas e mínimas diárias anuais, na região Alto Rio Grande, à montante da UHE de Camargos/CEMIG, verificando as possíveis relações com as características físicas e hidrogeológicas das bacias hidrográficas estudadas.

\section{MATERIAL E MÉTODOS}

\section{Caracterização Fisiográfica da região e séries históricas}

A região Alto Rio Grande, à montante da represa da UHE de Camargos/CEMIG, pertence à Bacia do Rio Grande, cuja nascente localiza-se junto à Serra da Mantiqueira, drenando até o reservatório da referida usina, por meio de dois rios principais Grande e Aiuruoca. Possui clima Cwb e Cwa, pela classificação de Köppen, com invernos frios, geadas frequentes, e verões brandos, com elevada concentração das chuvas no período de novembro a março. Sua geomorfologia apresenta relevo movimentado, com altitude média de $1000 \mathrm{~m}$. A vegetação varia de subformas de cerrado e campo cerrado, com florestas de Mata Atlântica e Araucária, próximo a Serra da Mantiqueira. Ressalta-se que Cambissolos e Solos Litólicos (Neossolos) preenchem $75 \%$ da área (Araújo, 2006; ; Junqueira Junior et al., 2008).

A região foi subdividida em seis bacias hidrográficas, com áreas variando de 105 a $2226 \mathrm{~km}^{2}$, conforme disponibilidade de estações fluviométricas. $\mathrm{Na}$
Tabela 1, apresentam-se as bacias, com respectivas áreas de drenagem, cursos d'água principal e respectivos comprimentos $(\mathrm{L})$, diferenças de cotas entre a cabeceira e a seção de controle $(\mathrm{H})$, índice de compacidade $(\mathrm{kc})$, sinuosidade do curso d'água principal (S) e declividade média, em $\mathrm{m} / \mathrm{km}$ de rio. Os dados de vazão diária foram obtidos junto ao banco de dados HIDROWEB/ANA e CEMIG, constituindo-se séries históricas de vazões máximas e mínimas diárias anuais, de 1969 a 2002, não havendo falha nas mesmas. Na Figura 1, apresenta-se o mapa com a localização da região no contexto estadual.

\section{Distribuições de Probabilidades e Intervalos de Confiança}

Confrontaram-se as frequências observadas das seis séries históricas de vazões máximas com as frequências estimadas pela Distribuição de Probabilidades Gumbel e as das séries de vazões mínimas pela distribuição Gama. As estimativas dos parâmetros foram realizadas com base em algoritmos da Máxima Verossimilhança, conforme descrito em Haan (2002).

Foram estimadas as vazões máximas, associadas aos tempos de recorrência de 20,50 e 100 anos e mínimas associadas às frequências de excedência de 95,90 e $85 \%$. Foram construídos os respectivos intervalos de confiança dos parâmetros das distribuições, conforme Casella \& Berger (1990), Martins \& Clarke (1993) e Beijo et al. (2005). Os valores obtidos foram analisados à luz das características fisiográficas (Tabela 1) e hidrogeológicas e pedológicas da região, extraídas de Araújo (2006).

A função geral de Verossimilhança de uma distribuição de probabilidades é dada por:

$$
\mathrm{L}\left(\theta ; \mathrm{x}_{1} \ldots \mathrm{x}_{\mathrm{n}}\right)=\prod_{\mathrm{i}=1}^{\mathrm{n}} \mathrm{f}\left(\mathrm{x}_{\mathrm{i}} ; \theta\right)
$$

em que:

$\theta$ é um vetor que representa o conjunto de parâmetros da função de probabilidades;

x é a variável hidrológica;

f é a distribuição de probabilidades.

Essa expressão significa que a verossimilhança de uma distribuição com q parâmetros é dada pelo produtório da função. A função logaritmo da verossimilhança da distribuição Gumbel e Gama, respectivamente, são dadas por:

$$
\begin{aligned}
& 1(u, b)=-n \cdot \operatorname{Ln}(b)+\sum_{i=1}^{n}\left[-\left(\frac{x i-u}{b}\right)\right]-\sum_{i=1}^{n} \exp \left[-\left(\frac{x i-u}{b}\right)\right] \\
& 1(\alpha, \beta)=-n \cdot \alpha \cdot \operatorname{Ln}(\beta)-n \cdot \operatorname{Ln}[\Gamma(\alpha)]+(\alpha-1) \cdot \operatorname{Ln}\left[\sum \operatorname{Ln}(x i)\right]-\frac{\sum x i}{\beta}
\end{aligned}
$$


Os parâmetros u e b (Gumbel) e a e b (Gama) podem ser obtidos conforme Casela \& Berger (1990) e Naghetinni $\&$ Pinto (2007).

Segundo Casella \& Berger (1990), a precisão das estimativas de Máxima Verossimilhança é obtida pelo inverso de uma matriz constituída pela esperança matemática das derivadas parciais, de segunda ordem, da função log-verossimilhança. Esta matriz é conhecida como Matriz de Informação de Fisher e é dada pela seguinte expressão:

$$
I(b, v)=\left[\begin{array}{ll}
-E\left(\frac{\partial^{2} l(b, v)}{\partial b^{2}}\right) & -E\left[\frac{\partial^{2} l(b, v)}{\partial b \partial v}\right] \\
-E\left[\frac{\partial^{2} l(b, v)}{\partial b \partial v}\right] & -E\left[\frac{\partial^{2} l(b, v)}{\partial v^{2}}\right]
\end{array}\right]
$$

em que:

I (b,v) é a Matriz de Informação de Fisher;

b e v são parâmetros de uma determinada distribuição de probabilidades.

Assim, obtendo-se a inversa de I, tem-se:

$$
\mathrm{W}=\mathrm{I}^{-1}(\mathrm{~b}, \mathrm{v})=\left[\begin{array}{cc}
\operatorname{var}(\mathrm{b}) & \operatorname{cov}(\mathrm{b}, \mathrm{v}) \\
\operatorname{cov}(\mathrm{v}, \mathrm{b}) & \operatorname{var}(\mathrm{v})
\end{array}\right]
$$

Essas concepções foram desenvolvidas com base no fato de que, quando o tamanho da série é grande o suficiente, os parâmetros estimados por Máxima Verossimilhança apresentam distribuição normal conjunta, possibilitando a geração dos intervalos de confiança. Para a distribuição Gumbel, as derivadas parciais de segunda ordem da função log-verossimilhança, e suas respectivas esperanças são dadas por:

$$
\begin{gathered}
\frac{\partial^{2} 1(\mathrm{u}, \mathrm{b})}{\partial \mathrm{u}^{2}}=\frac{1}{\mathrm{~b}^{2}} \cdot \sum_{\mathrm{i}=1}^{\mathrm{n}} \exp \left[-\left(\frac{\mathrm{xi}-\mathrm{u}}{\mathrm{b}}\right)\right] . \therefore-\mathrm{E}=\frac{\mathrm{n}}{\mathrm{b}^{2}} \\
\frac{\partial^{2} 1(\mathrm{u}, \mathrm{b})}{\partial \mathrm{b}^{2}}=\frac{\mathrm{n}}{\mathrm{b}^{2}}-\frac{2}{\mathrm{~b}^{2}} \cdot \sum_{\mathrm{i}=1}^{\mathrm{n}} \exp \left[\left(\frac{\mathrm{xi}-\mathrm{u}}{\mathrm{b}}\right)\right]+\frac{2}{\mathrm{~b}^{2}} \cdot \sum_{\mathrm{i}=1}^{\mathrm{n}}\left(\frac{\mathrm{xi}-\mathrm{u}}{\mathrm{b}}\right) \exp \left[-\left(\frac{\mathrm{xi}-\mathrm{u}}{\mathrm{b}}\right)\right]+\frac{1}{\mathrm{~b}^{2}} \cdot \sum_{\mathrm{i}=1}^{\mathrm{n}}\left(\frac{\mathrm{xi}-\mathrm{u}}{\mathrm{b}}\right)^{2} \times \\
\exp \left[-\left(\frac{\mathrm{xi}-\mathrm{u}}{\mathrm{b}}\right)\right] \cdot \therefore-\mathrm{E}=\frac{1,8237 \cdot \mathrm{n}}{\mathrm{b}^{2}} \\
\frac{\partial^{2} 1(\mathrm{u}, \mathrm{b})}{\partial \mathrm{u} \partial \mathrm{b}}=\frac{-\mathrm{n}}{\mathrm{b}^{2}}-\frac{1}{\mathrm{~b}^{2}} \cdot \sum_{\mathrm{i}=1}^{\mathrm{n}}\left(\frac{\mathrm{xi}-\mathrm{u}}{\mathrm{b}}\right) \cdot \exp \left[-\left(\frac{\mathrm{xi}-\mathrm{u}}{\mathrm{b}}\right)\right]+\frac{1}{\mathrm{~b}^{2}} \cdot \sum_{\mathrm{i}=1}^{\mathrm{n}} \exp \left[-\left(\frac{\mathrm{xi}-\mathrm{u}}{\mathrm{b}}\right)\right] . \therefore-\mathrm{E}=\frac{0,4228 \cdot \mathrm{n}}{\mathrm{b}^{2}}
\end{gathered}
$$

A inversa da matriz de Informação de Fisher, bem como o intervalo de confiança dos parâmetros, ficam definidos por:

$$
\begin{gathered}
\mathrm{W}=\mathrm{I}^{-1}(\mathrm{u}, \mathrm{b})=\left[\begin{array}{ll}
\frac{1,1087 \cdot \mathrm{b}^{2}}{\mathrm{n}} & \frac{0,257 \cdot \mathrm{b}^{2}}{\mathrm{n}} \\
\frac{0,257 \cdot \mathrm{b}^{2}}{\mathrm{n}} & \frac{0,6079 \cdot \mathrm{b}^{2}}{\mathrm{n}}
\end{array}\right] \\
\mathrm{IC}(\mathrm{u})=\mathrm{u} \pm \mathrm{z}_{\alpha / 2} \cdot \frac{1,0529 \cdot \mathrm{b}}{\sqrt{\mathrm{n}}} \mathrm{e} \\
\mathrm{IC}(\mathrm{b})=\mathrm{b} \pm \mathrm{z}_{\alpha / 2} \cdot \frac{0,7797 \cdot \mathrm{b}}{\sqrt{\mathrm{n}}}
\end{gathered}
$$

em que:

n é o tamanho da série histórica; z é a variável reduzida da distribuição normal padrão; $\alpha$ é a cobertura do intervalo $(a=5 \%)$.

Para a distribuição Gama, o intervalo de confiança de seus parâmetros pode ser estimado da seguinte forma:

$$
\frac{\partial^{2} 1(\alpha, \beta)}{\partial \alpha^{2}}=-\psi^{\prime}(\alpha) \Rightarrow-E=\psi^{\prime}(\alpha)
$$

Em que $\psi$ 'é a derivada da função digama.

$$
\begin{aligned}
& \frac{\partial^{2} 1(\alpha, \beta)}{\partial \alpha \partial \beta}=\frac{-1}{\beta} \Rightarrow-\mathrm{E}=\frac{1}{\beta} \\
& \frac{\partial^{2} 1(\alpha, \beta)}{\partial \beta^{2}}=\left(\frac{\alpha}{\beta^{2}}-\frac{2 \cdot \alpha \cdot \beta}{\beta^{3}}\right) \Rightarrow-\mathrm{E}=\frac{\alpha}{\beta^{2}}
\end{aligned}
$$


A inversa da Matriz de Informação de Fisher, bem como as expressões para o intervalo de confiança, são dadas por:

$$
\begin{aligned}
& \mathrm{W}=\mathrm{I}^{-1}(\alpha, \beta)=\mathrm{n} \cdot\left[\begin{array}{cc}
\psi^{\prime}(\alpha) & \frac{1}{\beta} \\
\frac{1}{\beta} & \frac{\alpha}{\beta^{2}}
\end{array}\right] \\
& \alpha=\alpha \pm z_{\alpha / 2} \cdot \frac{\alpha}{n \cdot\left[\alpha \cdot \psi^{\prime}(\alpha)-1\right]} \quad \text { e } \\
& \beta=\beta \pm \mathrm{z}_{\alpha / 2} \cdot \frac{\beta^{2} \cdot \psi^{\prime}(\alpha)}{\mathrm{n} \cdot\left[\alpha \cdot \psi^{\prime}(\alpha)-1\right]}
\end{aligned}
$$

$\mathrm{O}$ teste de $\mathrm{x}^{2}$ foi aplicado para avaliar a precisão do ajuste de ambas as distribuições de probabilidades e sua consequente adequação, pois segundo Naghettini \& Pinto (2007), este teste é um excelente indicador da precisão do ajuste por refletir o quadrado médio do erro. Para sua aplicação, busca-se agrupar os dados da série histórica em classes de frequência, acumulando-se os erros calculados entre a frequência observada na respectiva classe i $\left(f_{o j}\right)$ e a correspondente frequência teórica, calculada pela distribuição de probabilidades ajustada $\left(f_{t i}\right)$, ou seja:

$$
\lambda_{\text {calculado }}^{2}=\sum_{\mathrm{i}=1}^{\mathrm{n}} \frac{\left(\mathrm{f}_{\mathrm{oi}}-\mathrm{f}_{\mathrm{ti}}\right)^{2}}{\mathrm{f}_{\mathrm{ti}}}
$$

Para que a distribuição de probabilidades possa ser considerada adequada, o valor de $\lambda^{2}$ calculadao deve ser menor que o valor de $\lambda^{2}$ tabela ou seja, a hipótese $\mathrm{H}_{0}$ do teste não é rejeitada, o que significa que as frequências observadas podem ser modeladas pela distribuição de probabilidades em questão. Os graus de liberdade (v) para obtenção de $\lambda^{2}{ }_{\text {tabela }}$ foram calculados, segundo Naghettini \& Pinto (2007), por:

$$
\mathrm{v}=\mathrm{n}-\mathrm{k}-1
$$

em que:

$\mathrm{n}$ corresponde ao número de classes e $\mathrm{k}$ ao número de parâmetros da distribuição.

Tabela 1 - Características morfométricas das bacias hidrográficas estudadas.

\begin{tabular}{cccccccc}
\hline Estação Fluviométrica & Curso d'água & $\mathrm{A}\left(\mathrm{km}^{2}\right)$ & $\mathrm{H}(\mathrm{m})$ & $\mathrm{L}(\mathrm{km})$ & $\mathrm{kc}$ & $\mathrm{S}$ & $\mathrm{H} / \mathrm{L}(\mathrm{m} / \mathrm{km})$ \\
\hline Aiuruoca (1) & Rio Aiuruoca & 536 & 850 & 58,68 & 1,65 & 1,31 & 14,48 \\
Andrelândia (2) & Rio Turvo Pequeno & 274 & 199 & 41,95 & 1,52 & 1,40 & 4,74 \\
Bom Jardim (3) & Rio Grande & 509 & 675 & 92,43 & 2,00 & 1,62 & 7,30 \\
Carvalhos (4) & Ribeirão do Francês & 105 & 465 & 23,72 & 1,23 & 1,55 & 19,60 \\
F. Laranjeiras (5) & Rio Aiuruoca & 2083 & 860 & 156,4 & 1,44 & 1,86 & 5,50 \\
Madre de Deus (6) & Rio Grande & 2226 & 760 & 260,65 & 2,22 & 2,29 & 2,92 \\
\hline
\end{tabular}

$\mathrm{A}=$ área da bacia; $\mathrm{H}$ = desnível cabeceira - seção de controle; $\mathrm{L}=$ comprimento do curso d'água principal; Kc = índice de compacidade, $\mathrm{S}=$ sinuosidade. F. Laranjeiras = Fazenda Laranjeiras. Fonte: Silva et al. (2006).

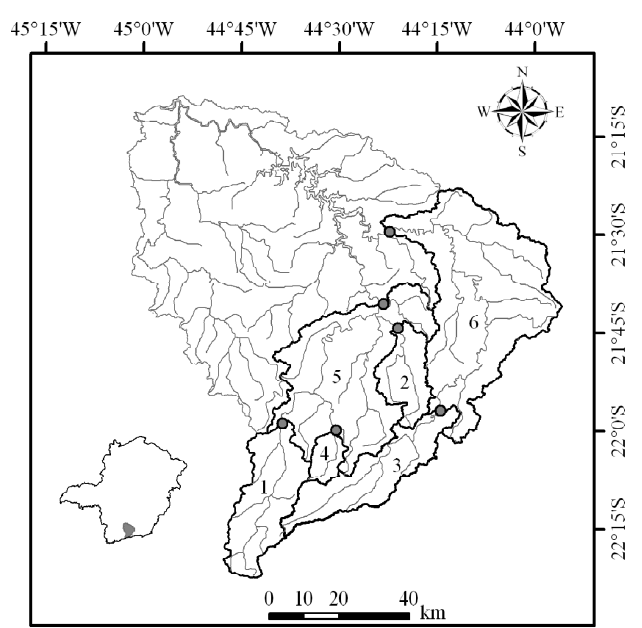

Figura 1 - Localização da região Alto Rio Grande à montante da UHE de Camargos e identificação de suas principais bacias. 


\section{RESULTADOS E DISCUSSÃO}

Na Tabela 2, apresentam-se os valores de $\mathrm{x}^{2}$ calculados para a distribuição Gumbel e Gama, respectivos valores de $\mathrm{x}^{2}$ tabelados, ao nível de significância de 5\% de probabilidade e adequação das distribuições. É possível observar que as distribuições aplicadas foram adequadas às respectivas séries históricas, com valores muito baixos de $\mathrm{x}^{2}$ calculados, refletindo a boa precisão dos ajustes das distribuições de probabilidades.

Na Tabela 3, apresentam-se os intervalos de confiança, valores de vazão máxima diária anual e rendimentos específicos, para os tempos de recorrência de 20, 50 e 100 anos. As bacias hidrográficas de Andrelândia, F. Laranjeiras e Madre de Deus apresentaram valores de rendimentos específicos máximos semelhantes para cada tempo de recorrência, isto é, aproximadamente $160 \mathrm{~L} / \mathrm{s} . \mathrm{km}^{2}$ para TR de 20 anos, $180 \mathrm{~L} / \mathrm{s} . \mathrm{km}^{2}$ para TR de 50 anos e $200 \mathrm{~L} / \mathrm{s} \mathrm{km}^{2}$ para recorrência de 100 anos. Esses valores permitem inferir que os efeitos produzidos por cheias históricas nestas bacias tendem a ser semelhantes. No caso específico de F. Laranjeiras e Madre de Deus, que apresentam áreas de drenagem próximas (Tabela 1), observam-se valores máximos para a primeira superiores aos da segunda, o que não era de se esperar em se tratando da magnitude do Rio Grande em relação ao Rio Aiuruoca. As diferenças se justificam pelas características morfométricas das bacias (Tabela 1), como comprimento do curso d'água principal, consideravelmente menor para um desnível maior entre as extremidades e índice de compacidade inferior, próximo a 1 , significando uma bacia com propensão a produzir cheias de maior intensidade.

Para a bacia Bom Jardim, os efeitos de uma cheia podem ser mais prejudiciais do que para as 3 bacias anteriores, uma vez que os rendimentos específicos máximos são superiores. No entanto, para as bacias de Carvalhos e Aiuruoca, especialmente nesta última, os efeitos podem ser ainda mais pronunciados, com valores acima de $340 \mathrm{~L} / \mathrm{s} . \mathrm{km}^{2}$. Observa-se que as bacias de Aiuruoca e Carvalhos apresentam grandes desníveis para cursos d'água com pequeno comprimento. Essa situação, aliada ao comportamento da sinuosidade (1,31 e 1,55 respectivamente), caracterizam cursos d'água como propensos a cheias de maior magnitude. Além disso, verificam-se índices de compacidade baixos o suficiente para caracterizá-los como de propensão a grandes cheias.

Observa-se, na Tabela 3, que os intervalos de confiança para as bacias hidrográficas de Madre de Deus, Fazenda Laranjeiras e Andrelândia são inferiores aos obtidos para Aiuruoca, Carvalhos e Bom Jardim, especialmente as duas primeiras. Segundo Katz et al. (2002) e George (2007), isto se explica pelas características fisiográficas das bacias, onde suas declividades também são consideravelmente maiores, mostrando maior influência topográfica no escoamento de cheia, o que inevitavelmente produz maior variabilidade das vazões máximas, fazendo com que o intervalo de possíveis valores seja maior.

Analisando-se ainda os intervalos de confiança na Tabela 3, observam-se valores semelhantes para as bacias de Andrelândia, F. Laranjeiras e Madre de Deus (de 30 a 40 $\mathrm{L} / \mathrm{s} . \mathrm{km}^{2}$ ), e valores mais elevados para Aiuruoca e Carvalhos (de 70 a 99 L/s.km²). Trabalhando com bacias hidrográficas de porte semelhante as deste trabalho e com a distribuição Gumbel, Martins \& Clarke (1993), estimaram intervalos de confiança, pela metodologia da máxima verossimilhança, com $95 \%$ de cobertura, obtendo amplitudes semelhantes às obtidas neste trabalho. No entanto, os mesmos não relacionaram os resultados às características fisiográficas das bacias para validação e interpretação dos resultados. Essas informações são fundamentais para subsidiar o manejo das bacias no tocante a aspectos de drenagem superficial, ou seja, os critérios de dimensionamento poderão ser semelhantes nas 3 primeiras bacias, enquanto que nas bacias de Bom Jardim, Carvalhos e Aiuruoca, os mesmos deverão ser mais rigorosos.

Tabela 2 - Valores de Qui-quadrado calculados e tabelados para séries históricas de vazões máxima e mínima diária anual e respectiva adequação estatística.

\begin{tabular}{ccccc}
\hline Estação & \multicolumn{2}{c}{ Vazão Máxima Diária Anual } & \multicolumn{2}{c}{ Vazão Mínima Diária Anual } \\
\cline { 2 - 5 } Fluviométrica & Gumbel & Tabela & Gama & Tabela \\
\cline { 2 - 5 } Aiuruoca & $3,423 \mathrm{~A}$ & 9,49 & $0,297 \mathrm{~A}$ & 5,99 \\
Andrelândia & $4,048 \mathrm{~A}$ & 7,81 & $3,940 \mathrm{~A}$ & 5,99 \\
Bom Jardim & $0,115 \mathrm{~A}$ & 7,81 & $0,899 \mathrm{~A}$ & 5,99 \\
Carvalhos & $0,191 \mathrm{~A}$ & 7,81 & $1,100 \mathrm{~A}$ & 5,99 \\
F. Laranjeiras & $1,052 \mathrm{~A}$ & 5,99 & $0,954 \mathrm{~A}$ & 5,99 \\
Madre de Deus & $1,655 \mathrm{~A}$ & 3,84 &
\end{tabular}

$\mathrm{A}=$ adequado. 
Tabela 3 - Vazões máximas e rendimentos específicos máximos para bacias hidrográficas da região Alto Rio Grande à montante da represa da UHE de Camargos/CEMIG.

\begin{tabular}{cccc}
\hline Estação Fluviométrica & TR (anos) & $\mathrm{Q}\left(\mathrm{m}^{3} / \mathrm{s}\right)$ & $\mathrm{RE}\left(\mathrm{L} / \mathrm{s} \cdot \mathrm{km}^{2}\right)$ \\
\hline \multirow{3}{*}{ Aiuruoca } & 20 & $183 \pm 38,44$ & $342,39 \pm 71,72$ \\
& 50 & $212,73 \pm 46,73$ & $396,88 \pm 87,18$ \\
& 100 & $234,62 \pm 52,94$ & $437,72 \pm 98,77$ \\
\hline \multirow{2}{*}{ Andrelândia } & 20 & $45,85 \pm 8,12$ & $167,34 \pm 29,64$ \\
& 50 & $52,23 \pm 9,87$ & $190,62 \pm 36,02$ \\
Bom Jardim & 100 & $57,01 \pm 11,18$ & $208,07 \pm 40,80$ \\
\hline \multirow{3}{*}{ Carvalhos } & 20 & $96,96 \pm 18,62$ & $190,49 \pm 36,58$ \\
& 50 & $111,59 \pm 22,64$ & $219,23 \pm 44,48$ \\
& 100 & $122,55 \pm 25,64$ & $240,77 \pm 50,37$ \\
\hline \multirow{2}{*}{ F. Laranjeiras } & 20 & $30,99 \pm 5,76$ & $295,14 \pm 54,86$ \\
& 50 & $35,52 \pm 7,00$ & $338,29 \pm 66,67$ \\
& 100 & $38,91 \pm 7,93$ & $370,57 \pm 75,52$ \\
\hline \multirow{2}{*}{ Madre de Deus } & 20 & $349,11 \pm 60,86$ & $156,83 \pm 27,34$ \\
& 50 & $396,93 \pm 73,99$ & $178,32 \pm 33,24$ \\
& 100 & $432,76 \pm 83,82$ & $194,41 \pm 37,65$ \\
\hline
\end{tabular}

$\mathrm{RE}=$ rendimento específico; $\mathrm{TR}=$ tempo de recorrência.

Na Tabela 4, apresentam-se os valores de vazões mínimas e respectivos rendimentos específicos mínimos e seus intervalos de confiança. Uma análise importante desses dados corresponde à frequência de excedência de $90 \%$, a qual é referência para gestão dos recursos hídricos superficiais. Para a bacia de Aiuruoca, o mesmo varia de 8,302 a 10,802 L/s.km², correspondendo aos maiores valores obtidos. Na sequência, tem-se a bacia de Carvalhos, com valores entre 7,810 e 10,286 L/s. $\mathrm{km}^{2}$, sendo as duas bacias com maior disponibilidade hídrica. Em estudo pedológico e hidrológico recente, desenvolvido por Araújo (2006) na região, foi constatado que a geologia destas bacias é constituída por mantos de alteração caracterizados por depósitos de fluxo gravitacional de detritos espessos, com bom potencial de armazenamento de águas subterrâneas. Há predomínio de gnaisses de alto grau de metamorfismo, granitóides e quartzitos. Assim, em razão dessas características hidrogeológicas, as quais regem o comportamento dos aquíferos, essas bacias apresentam maior potencial para restituição do escoamento subterrâneo no período seco do ano, proporcionando vazões mais significativas. Em termos de estimativa dos valores desta variável, Silva et al. (2006), ajustando a distribuição Gumbel pelo método dos momentos, obtiveram resultados dentro dos intervalos de confiança, mas, na maioria das vezes, próximos do limite superior do intervalo, mostrando que é necessário aprofundar nas análises associadas à inferência estatística para distribuições assimétricas, uma vez que a aplicação dos resultados pode ficar comprometida.

As bacias de Madre de Deus, F. Laranjeiras e Andrelândia apresentaram os menores valores para o rendimento específico mínimo, variando de 5,211 a 6,721 L/ s.km², 7,009 a 9,025 L/s.km² e 6,460 a 8,358 L/s. $\mathrm{km}^{2}$, respectivamente. Com base nesses resultados, verifica-se que a vazão outorgável nessas bacias será inferior às bacias mencionadas anteriormente. Este comportamento também encontra base científica no estudo de Araújo (2006), onde, nesta região, especialmente em F. Laranjeiras, encontra-se o domínio dos Cambissolos de rocha pelítica (textura fina), com menor espessura do manto de alteração. A pequena espessura do solo, aliada ao relevo movimentado da região, indicam que o armazenamento de água subterrânea ocorre apenas na parte do manto composto por rocha argilosa, com baixa condutividade 
Tabela 4 - Vazões mínimas e rendimentos específicos mínimos para bacias hidrográficas da região do Alto Rio Grande à montante da represa da UHE de Camargos/CEMIG.

\begin{tabular}{cccc}
\hline Estação Fluviométrica & F. Excedência & $\mathrm{Q}\left(\mathrm{m}^{3} / \mathrm{s}\right)$ & $\mathrm{RE}\left(\mathrm{L} / \mathrm{s} \cdot \mathrm{km}^{2}\right)$ \\
\hline \multirow{2}{*}{ Aiuruoca } & $95 \%$ & $4,60 \pm 0,61$ & $8,582 \pm 1,138$ \\
& $90 \%$ & $5,12 \pm 0,67$ & $9,552 \pm 1,250$ \\
Andrelândia & $85 \%$ & $5,51 \pm 0,69$ & $10,280 \pm 1,287$ \\
\hline \multirow{2}{*}{ Bom Jardim } & $95 \%$ & $1,85 \pm 0,24$ & $6,752 \pm 0,876$ \\
& $90 \%$ & $2,03 \pm 0,26$ & $7,409 \pm 0,949$ \\
& $85 \%$ & $2,16 \pm 0,26$ & $7,883 \pm 0,949$ \\
\hline \multirow{2}{*}{ Carvalhos } & $95 \%$ & $4,37 \pm 0,57$ & $8,585 \pm 1,120$ \\
& $90 \%$ & $4,77 \pm 0,57$ & $9,371 \pm 1,120$ \\
& $85 \%$ & $5,05 \pm 0,60$ & $9,921 \pm 1,179$ \\
\hline \multirow{2}{*}{ F. Laranjeiras } & $95 \%$ & $0,84 \pm 0,10$ & $8,000 \pm 0,952$ \\
& $90 \%$ & $0,95 \pm 0,13$ & $9,048 \pm 1,238$ \\
& $85 \%$ & $1,03 \pm 0,13$ & $9,810 \pm 1,239$ \\
\hline & $95 \%$ & $15,20 \pm 2,00$ & $7,297 \pm 0,960$ \\
Madre de Deus & $90 \%$ & $16,70 \pm 2,10$ & $8,017 \pm 1,008$ \\
& $85 \%$ & $17,70 \pm 2,20$ & $8,497 \pm 1,056$ \\
\hline
\end{tabular}

F. Excedência = Freqüência de Excedência.

hidráulica. Dessa forma, pode-se afirmar que as bacias hidrográficas de Aiuruoca e Bom Jardim são as que podem produzir os maiores escoamentos subterrâneos ao longo do ano, podendo-se inferir que são bacias com maior disponibilidade de água no período seco do ano na região.

\section{CONCLUSÕES}

A construção dos intervalos de confiança das séries de vazões máximas e mínimas mostrou-se compatível com as características físicas da região (fisiográficas e hidrogeológicas), permitindo melhor caracterização do comportamento hidrológico da mesma. Com base nesses resultados, ficou evidenciado que as bacias hidrográficas de Aiuruoca, Bom Jardim e Carvalhos são as que produziram os maiores rendimentos específicos máximos e mínimos, sendo as áreas com maior potencial para geração de cheias mais significativas e maior disponibilidade hídrica no período seco do ano, apresentando comportamento hidrológico semelhante. As bacias hidrográficas de Madre de Deus, Fazenda Laranjeiras e Andrelândia são as que produziram os menores valores de vazões mínimas, sendo as áreas com menor disponibilidade hídrica.

\section{REFERÊNCIAS BIBLIOGRÁFICAS}

ARAÚJO, A.R. Levantamento de solos na Bacia do Alto Rio Grande: base para estudos hidrológicos e aptidão agrícola das terras. 2006. 190f. Tese (Doutorado em Ciência do Solo)-Universidade Federal de Lavras, Lavras, 2006.

BEIJO, L.A.; MUNIZ, J.A.; CASTRO NETO, P. Tempo de retorno das precipitações máximas em Lavras (MG) pela distribuição de valores extremos do tipo I. Revista Ciência e Agrotecnologia, Lavras, v.29, n.3, p.657-667, 2005.

\section{CASELLA, G.; BERGER, R.L. Statistical inference.} Belmont: Duxbury, 1990. 650p.

DOUGLAS, E.M.; VOGEL, R.M. Probabilistic behavior of floods of record in the United States. Journal of Hydrologic Engineering, Reston, v.11, n.5, p.482-488, 2006.

GEORGE, S. St. Streamflow in the Winnipeg River Basin, Canada: trends, extremes and climate linkages. Journal of Hydrology, Amsterdam, v.332, n.3/4, p.396-411, 2007. 
HAAN, C.T. Statistical methods in hydrology. 2.ed. Ames: The Iowa State University, 2002. 377p.

JÚNIOR, J. A. J.; SILVA, A. M. da; MELLO, C. R. de; PINTO, D. B. F. Continuidade espacial de atributos Físico-Hídricos do solo em sub-bacia hidrográfica de cadeceira. Ciência e Agrotecnologia, Lavras, v. 32, n. 3, p. 914 - 922, maio/jun., 2008.

KATZ, R.W.; PARLANGE, M.B.; NAVEAU, P. Statistics of extremes in hydrology. Advances in Water Resources, Amsterdam, v.25, p.1287-1304, 2002.

MAKI, A.; KENJI, T.; KIYOKAZU, K.; TERUO, H.

Morphological and physico-chemical characteristics of soils in a steppe region of the Kherlen River Basin, Mongolia.

Journal of Hydrology, Amsterdam, v.333, n.1, p.100-108, 2007.

MARTINS, E.S.P.R.; CLARKE, R.T. Likelihood-based confidence intervals for estimating floods with given return periods. Journal of Hydrology, Amsterdam, v.147, n.1, p.61-81, 1993.
MELLO, C.R. de; OLIVEIRA, G.C.; FERREIRA, D.F.; LIMA, J.M. Predição da porosidade drenável e da disponibilidade total de água para Cambissolos da região Campo das Vertentes. Pesquisa

Agropecuária Brasileira, Brasília, v.37, n.9, p.13191324, 2002.

NAGHETTINI, M.; PINTO, E.J.A. Hidrologia estatística. Belo Horizonte: CPRM, 2007. 552p.

QUEIROZ, M.M.F. de; CHAUDHRY, F.H. Análise de eventos hidrológicos extremos usando a distribuição GEV e momentos LH. Revista Brasileira de Engenharia Agrícola e Ambiental, Campina Grande, v.10, n.2, p.381389, 2006.

SILVA, A.M. da; OLIVEIRA, P.M.; MELLO, C.R. de; PIERANGELI, C. Vazões mínimas e de referência para outorga na região do Alto Rio Grande, $\mathrm{MG}$. Revista Brasileira de Engenharia Agrícola e Ambiental, Campina Grande, v.10, n.2, p.374-380, 2006. 